\title{
Immunization of Children: Knowledge, Attitude and Practice of Care-givers
}

\author{
Habiba Jiri-Musa ${ }^{1, *}$, Gidado Abubakar Auta ${ }^{2}$, Bobga Bala Ishaya ${ }^{3}$, Arziki Simeon Zoaka ${ }^{4}$, \\ Lumsami Shadrach ${ }^{5}$ \\ ${ }^{1}$ Primary Health Care Center, Premiere Urgence Internationale, Maiduguri, Nigeria \\ ${ }^{2}$ Department of Radiology, University of Maiduguri Teaching Hospital, Nigeria \\ ${ }^{3}$ Department of Internal Medicine, General Hospital Garkida, Nigeria \\ ${ }^{4}$ Medical and Dental Council of Nigeria, Nigeria \\ ${ }^{5}$ Department of Internal Medicine, Federal Medical Centre, Yola, Nigeria
}

Copyright $₫ 2018$ by authors, all rights reserved. Authors agree that this article remains permanently open access under the terms of the Creative Commons Attribution License 4.0 International License

\begin{abstract}
Vaccine preventable diseases are the commonest cause of mortality in children under five years of age. Therefore, immunizing a child significantly reduces cost of treating diseases and subsequently provides a healthy childhood. The objective of this study was to assess the knowledge, attitude and practice of caregivers to immunization of children under five years of age. This study was conducted over a period of four weeks with four hundred interviewer-based questionnaires given to our respondents, in a College Hospital at the North Eastern region of Nigeria. A cross sectional descriptive study was carried out using convenience sampling method. The data obtained was analyzed using Epi info software. The result of the study showed that $63 \%$ of children were fully immunized while $27 \%$ were either partially vaccinated or not vaccinated at all. It also showed that $72.9 \%$ of children delivered in the hospital were fully vaccinated as against the $35.2 \%$ of children who were delivered at home. A major concern that affects vaccination practices is the requirement for good storage and transportation of vaccines. Implementation of policies that will encourage both hospital delivery and strict adherence to the immunization schedule is an important step towards achieving adequate immunization.
\end{abstract}

Keywords Care-givers, Children, Immunization, Maiduguri, Under-five, Vaccination

\section{Introduction}

Immunization is one of the most powerful and cost-effective weapons of modern medicine as affirmed by the World Health Organization [1]. Immunization process involves inducing immunologic defense against infectious organisms without significant risk to the recipient. Immunization can prevent infectious disease in the individual, restrict the spread of the disease and may ultimately eradicate it in the community. Modern developments in immunization started in 1796 when Edward Jenner demonstrated experimentally the protective effect of cowpox extracts against small-pox with the inoculation of a person with relatively harmless disease material. He called the process of the inoculation "vaccination", derived from the Latin name of cow vaccinia [2].

It is however tragic that immunization services have been under-utilized in the world today. Immunizing a child significantly reduces cost of treating diseases, thus providing a healthy childhood and reducing poverty and suffering [3]. In the past few decades, immunization coverage rates have improved sufficiently in the developed countries, thereby conferring herd immunity, whereas most of the developing countries are still struggling with faltering rates [4].

Under-five mortality rate is defined as death of children under five years of age per 1000 live births. The under-five mortality rate in Nigeria was 143 per 1000 live births according to a World Bank report published in 2012 [5], which is considerably high as compared to figures obtainable in the developed countries and even though recent data revealed a drop in the under-five mortality rate, Nigeria is still among the top ten countries with the highest infant mortality rate [6]. In Nigeria, the high incidence of under-5 mortality is not unconnected with the fact that the routine immunization coverage is very low [7], hence the need to assess the knowledge, attitude and practice of care-givers to immunization of children under five.

Currently, vaccines used for immunization are administered in much the same way as Jenner's, but such materials are produced in very specialized processes, and 
the products are eventually largely safe for administration. The antigenic material could be live attenuated in which the actual causative factor of the disease is live but weakened in virulence and hence harmless, or killed or inactivated vaccines in which the causative factor is killed and only its substance is needed to initiate immune response in the person [8].

One of the first recorded evidence of immunization was in the $15^{\text {th }}$ century B.C. in China when dried small pox crust were introduced intra-nasally, and the practice of injecting pus from small pox pustule of a sick person into a cut on the hand of a healthy person (variolation) was said to be discovered in India [9].

Small pox has been one of human kind's greatest scourges since time immemorial. It is believed to have appeared at the time of the first Agricultural settlements in North Eastern Africa around 10,000 B.C. It probably spread from Africa to India by means of Egyptian merchants in the last millennium B.C. Small pox was described in China as early as in 1122 B.C. It was the desperate pressure to escape from this disease that lead to one of greatest breakthrough in Medicine, 3 centuries later and subsequently, the eradication of one of the greatest scourges of mankind. This process was termed variolation and started in China and spread to Europe, becoming the foundation for modern scientific immunology. The primary side effect of variolation was the appearance of small pox itself [2, 9]. Modern developments in immunization started in 1796 by Edward Jenner.

\section{Justification for the Study}

Since the under-five mortality in Nigeria is high and most deaths are caused by vaccine preventable diseases, it is importance to access the knowledge attitude and practice of caregivers of children under five years, with the aim of using the information in planning and improvement of immunization coverage.

\section{The Significance of This Study}

Assessing the knowledge, attitude and practice of care-givers to immunization will help identify specific problems peculiar to the community studied and further help in planning strategies to adopt in overcoming immunization setbacks and thus the need for this study.

\section{The Objectives of This Study}

1. To determine the knowledge of care-givers to immunization in a college Hospital in the Northern part of Nigeria.

2. To determine the attitude of care-givers towards immunization.

3. To identify the factors that influences the practice of care-givers towards immunization.
4. To determine the effectiveness of BCG vaccine by assessing the BCG scar among under-five.

\section{Materials and Methods}

This study was carried out to assess the perception of care-givers to immunization of children under 5 years attending a college Hospital in the Northern part of Nigeria, over a two months period. Respondents were selected on the basis of clinic attendance within the data collection period. Approval was sort and granted by the hospital management.

\subsection{Locale}

The college Hospital is a third-generation hospital and at the time of the study had a capacity of twenty three wards with bed capacity of over 540 beds and total of 17 clinical and 14 non-clinical departments. This study was carried out in the pediatric department of the hospital which had 3 wards (special Care Baby Unit, Emergency Pediatric Unit and the Pediatric Medical ward) and 6 units. It had a total number of about 86 beds. Clinics were conducted on various days of the week, Monday being SCBU clinic, Tuesday team C, Wednesday team A, Thursday team B and Friday team $\mathrm{D}$. The Well baby clinic where immunization based on the NPI schedule are on Tuesdays and Fridays.

\subsection{Study Design}

The study was a cross-sectional descriptive type. It included all children under five years. The tool for the study was the questionnaire. Responses were recorded using interviewer administered questionnaire.

\subsection{Sample Size Estimation}

The sample size was estimated using the formula

$$
\mathrm{n}=\frac{\mathrm{Z}^{2} \mathrm{Pq}}{\mathrm{d}^{2}}
$$

where $\mathrm{n}=$ Minimum sample required

$\mathrm{Z}=$ standard normal deviation (1.96)

$\mathrm{P}=$ anticipated proportion of children who were completely immunized $=0.5$

$$
\mathrm{q}=1-\mathrm{P}=0.5
$$

$\mathrm{d}=$ standard error precision $=0.05$

$$
\text { Hence } \mathrm{n}=\frac{1.96^{2} \times 0.5 \times 0.5}{\left(0.05^{2}\right)}=384.16=384.16
$$

$384.16 \approx 400$ (to make room for none response and wastages). Thus, the minimum sample size was 400 . This minimum sample size was used for the study.

\subsection{Sampling method}

Convenience sampling method was used for the study. A 
questionnaire was designed and after informed consent, was allocated to care-givers attending the well-baby clinic, out-patient pediatric clinic and those in the pediatric ward. A sample unit being a child that less than or equal to five years. A child is selected by chance based on availability, cooperation and convenience of the care giver.

\section{Results and Discussion}

The data obtained from the questionnaire were computed and analyzed using Epi Info software. The study was carried out in the pediatric unit of a college Hospital in Northern Nigeria, where a total of 400 questionnaires were administered, analyzed and discussed. There was a 100\% response rate.

The knowledge, attitude and practice of care-givers towards immunization, overall immunization coverage, presence of BCG scar, and the mother's Tetanus toxoids immunization status were studied. Similarly, the effect of such factors as the demographic characteristics of the care-givers, were also analyzed.

Table 1. Socio-demographic characteristics of respondents

\begin{tabular}{|c|c|c|}
\hline AGE (YEARS) & FREQUENCY & PERCENTAGE (\%) \\
\hline$<15$ & 4 & 1.0 \\
\hline $15-24$ & 157 & 39.3 \\
\hline $25-39$ & 225 & 56.2 \\
\hline$>40$ & 14 & 3.5 \\
\hline TOTAL & 400 & 100 \\
\hline \multicolumn{3}{|c|}{ RELATIONSHIP OF CAREGIVER TO CHILD } \\
\hline Mother & 370 & 92.5 \\
\hline Father & 6 & 1.5 \\
\hline Aunt & 17 & 4.3 \\
\hline Guardian & 5 & 1.3 \\
\hline Others & 2 & 0.5 \\
\hline TOTAL & 400 & 100 \\
\hline \multicolumn{3}{|c|}{ EDUCATIONAL STATUS } \\
\hline Primary & 35 & 8.8 \\
\hline Secondary & 114 & 28.5 \\
\hline Post-secondary & 163 & 40.8 \\
\hline Qur'anic & 61 & 15.3 \\
\hline TOTAL & 400 & 100 \\
\hline \multicolumn{3}{|c|}{ OCCUPATION } \\
\hline Civil servants & 76 & 19.0 \\
\hline Nurses & 11 & 2.8 \\
\hline Traders & 43 & 10.8 \\
\hline $\begin{array}{c}\text { Stay at home } \\
\text { parent }\end{array}$ & 213 & 53.3 \\
\hline Others & 57 & 14.3 \\
\hline TOTAL & 400 & 100 \\
\hline
\end{tabular}

\subsection{Demographic Characteristics of the Respondents}

Table 1 showed a greater number 382 (95.5\%) of the care-givers were between ages 15-39 years and only 4 respondents $(1.0 \%)$ were below 15years, while those above 40 years were only 14 (3.5\%) of the respondents. Majority $370(92.5 \%)$ of the care-givers were mothers of the children. Only $6(1.5 \%)$ of the respondent were fathers. About half 213 (53.3\%) of the respondents were stay at home mums and 87 (21.8\%) were civil servants (including nurses). There were 43 traders (10.8\%) and the remaining were others, most of which were students. Two third 277 (69.3\%) of our respondents had formal education beyond secondary school level. This may relate to the high rate (63\%) of children fully vaccinated in our study. It has been documented by Tadesse et al., 2009 and Breiman et al., 2004 that children born to mothers with secondary and higher level of education are twice more likely to receive full immunization than children born to mothers with no education $[10,11]$. This minimum sample size was used for the study.

Table 2. Place of delivery, immunization status and BCG scar

\begin{tabular}{|c|c|c|}
\hline $\begin{array}{c}\text { PLACE OF } \\
\text { DELIVERY }\end{array}$ & FREQUENCY & $\begin{array}{c}\text { PERCENTAGE } \\
\mathbf{( \% )}\end{array}$ \\
\hline Hospital & 295 & 73.8 \\
\hline Home & 105 & 26.3 \\
\hline TOTAL & $\mathbf{4 0 0}$ & $\mathbf{1 0 0}$ \\
\hline \multicolumn{2}{|c|}{ IMMUNIZATION STATUS } \\
\hline Fully vaccinated & 252 & 63.0 \\
\hline $\begin{array}{c}\text { Partially } \\
\text { vaccinated }\end{array}$ & 103 & 25.8 \\
\hline Not vaccinated & 45 & 11.3 \\
\hline TOTAL & $\mathbf{4 0 0}$ & $\mathbf{1 0 0}$ \\
\hline \multicolumn{2}{|c|}{ BCG SCAR } & 75.8 \\
\hline BCG scar seen & 269 & 24.2 \\
\hline BCG scar not seen & 86 & $\mathbf{1 0 0}$ \\
\hline TOTAL & $\mathbf{3 5 5}$ & \\
\hline
\end{tabular}

\subsection{Immunization History and BCG Scarring}

Table 2 shows that 295 (73.8\%) of the children were delivered in the hospital, while 105 (26.3\%) were delivered at home. About two third 252(63\%) of the children were fully vaccinated, 103 (25.8\%) were partially vaccinated and $45(11.3 \%)$ were not vaccinated. This shows an improvement in immunization coverage as compared with the NDHS 2008 in which only 23\% of children under 5years were fully immunized [12]. This could be because this study was carried out in a tertiary center as compared to NDHS which is a national survey. From the data collected, 355 had BCG vaccine. Only 269 (75.8\%) of those who claimed to have had BCG vaccine had evidence of scar. The remaining 86 (24.2\%) had no scar and this can 
be attributed to the lack of potency of the vaccine, immune status of the recipient and technical error in administration. Similar trend was reported by Dhanawade et al. [13], with $91.4 \%$ visible scarring from a sample of 70 infants. This indicates a rise in the percentage of children with BCG scars, from the study when compared with previous studies.

\subsection{Effect of Delivery Location on Vaccination Status}

The location of child delivery at home or at a hospital is a factor of influence on the child's vaccination status. This likelihood is assessed on the possibility of a full, partial or no vaccination status within the sample space of this study.

From Table 3, about three quarters, 73.8\% (295) of the children were delivered in the hospital while the remaining quarter, 26.25\% (105) was at home. Of those delivered in the hospital, $72.9 \%$ (215) of them were fully vaccinated while $29.1 \%$ (80) were not fully vaccinated. For those delivered at home (105), less than half, 35.2\% (37) had full vaccination and a large proportion, 64.8\% (68) were not fully vaccinated. Similar previous work by Ahluwalia et al. had $68 \%$ of hospital delivered children fully vaccinated against $52 \%$ of home delivered children that were fully vaccinated [14]. It can be deducted that children born in the hospital were more likely to have been fully vaccinated, than those born at home.

\subsection{Care-givers Knowledge, Attitude and Practices}

Table 3. Relationship between place of delivery and immunization status

\begin{tabular}{|c|c|c|c|}
\hline PLACE OF DELIVERY & FULLY VACCINATED & NOT FULLY VACINATED & TOTAL \\
\hline Hospital & $215(72.9 \%)$ & $80(29.1 \%)$ & $295(100 \%)$ \\
\hline Home & $37(35.2 \%)$ & $68(64.8 \%)$ & $105(100 \%)$ \\
\hline TOTAL & $\mathbf{2 5 2 ( 6 3 \% )}$ & $\mathbf{1 4 8}(\mathbf{3 7 \%})$ & $\mathbf{4 0 0}(\mathbf{1 0 0} \%)$ \\
\hline
\end{tabular}

Table 4. Care-givers perception towards immunization.

\begin{tabular}{|c|c|c|c|c|}
\hline STATEMENT & AGREE & INDIFFERENT & DISAGREE & TOTAL \\
\hline $\begin{array}{c}\text { Immunization protects against all forms of } \\
\text { diseases }\end{array}$ & $333(80.3 \%)$ & $41(10.3 \%)$ & $26(6.5 \%)$ & 400 \\
\hline Infertility can occur due to immunization & $59(14.8 \%)$ & $39(9.8 \%)$ & $302(75.5 \%)$ & 400 \\
\hline Immunization causes fever and prolong ill-health & $39(9.8 \%)$ & $30(7.5 \%)$ & $331(82.8 \%)$ & 400 \\
\hline Immunization prevents a child from growing & $4(1 \%)$ & $25(6.3 \%)$ & $371(92.8 \%)$ & 400 \\
\hline Immunization can harm your child & - & $29(7.3 \%)$ & $371(92.8 \%)$ & 400 \\
\hline
\end{tabular}

Table 5. Tetanus toxoid (TT) immunization schedule

\begin{tabular}{|c|c|}
\hline Tetanus toxoid (TT) Period & Stage of administration and duration of protection. \\
\hline TT1 & At first contact or early pregnancy. \\
\hline TT2 & Four weeks after TT1. Protects for three years. \\
\hline TT3 & Six months after TT2 or during subsequent pregnancy. Protects for five years. \\
\hline TT4 & A year after TT3 or during subsequent pregnancy. Protects for 10 years. \\
\hline TT5 & One year after TT4 or during subsequent pregnancy. Protects for life. \\
\hline
\end{tabular}

The general perception of care-givers was assessed with regards to their response to the survey questions. The result of this is presented in Table 4.

Table 4 shows good attitude of care-givers to immunization of children under five years except that most of the care-givers $333(80.3 \%)$ are of the view that immunization protects against all forms of diseases. About 41 (10.3\%) are indifferent and only 26 (6.5\%) disagree. A total of $302(75.5 \%)$ of the care-givers disagree that immunization causes infertility and 331 (82.2\%) disagree that immunization causes fever and prolong ill-health. Majority of the care-givers 371 (92.8\%) disagree that immunization prevents a child from growing and 371 92.8\%) of our respondents also disagree that mmunization can harm their children. This shows that ost of our respondents have good attitude towards munization. This could be attributed to the fact that the (69.3\%) had some form of formal

\subsection{Tetanus Toxoid Immunization Status}

Table 5 shows the TT schedule from the Nigerian Federal Ministry of Health (Primary Healthcare Department). The distribution of tetanus toxoid (TT) immunization status of mothers assessed in this study is then presented in Figure 1. 


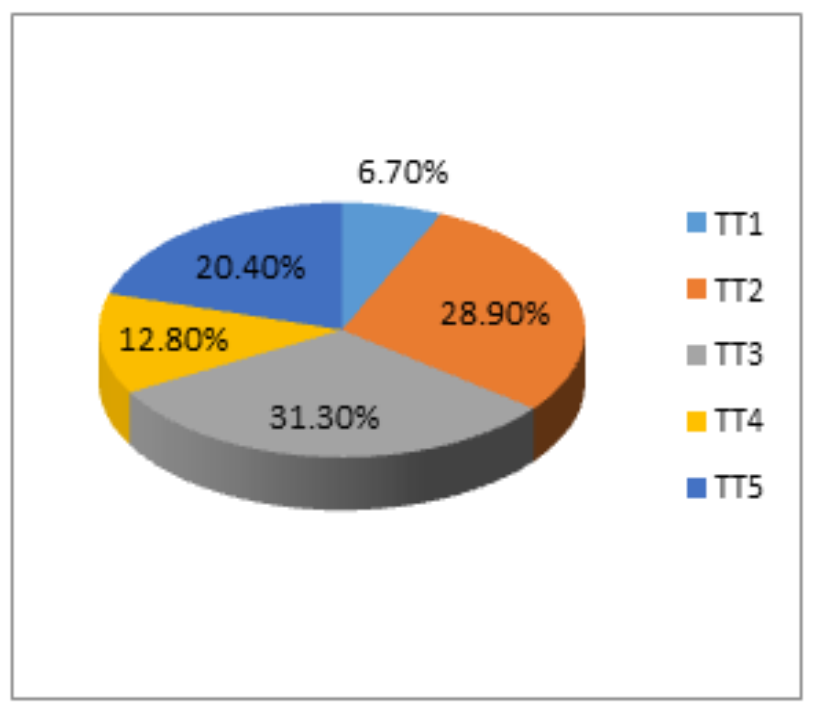

Figure 1. Mothers' Tetanus toxoid immunization status

Figure 1 shows 22 (6.7\%) of our respondents had only TT1, 95 (28.9\%) had TT2 and 103 (31.3\%) had TT3. Those having T4 are $42(12.8 \%)$ and 67 (20.4\%) completed their TT vaccine (TT5). About two - third (64.5\%) of the women who received TT vaccine had at least TT3.A similar study was conducted in Peshawar (Pakistan) by Naeem et al in which $55.6 \%$ of the respondents completed their TT vaccine (TT5) [15]. This figure is higher than the value obtained in our study (20.4\%). This may be a reason why neonatal tetanus is still prevalent in this environment.

\section{Conclusions}

As immunization is one of the most powerful and cost-effective weapons of modern medicine as affirmed by the WHO [1], this study will go a long way in identifying specific problems perculiar to the community and further help in planning strategies to adopt in overcoming immunization setbacks.

Information regarding the demographic characteristics of the caregiver, child immunization history either from the immunization card or the care giver, the presence or absence of a BCG scar, mothers' tetanus toxoid immunization status and the effects of factors such as caregivers' perception towards immunization were analysed. The results obtained were computed and analyzed critically using Epi info software and the following observations and results were arrived at;

- Majority of the respondents (69.3\%) had formal education beyond secondary school level. There was an overall positive impact of caregivers education on immunization coverage level.

- The percentage of children fully immunized was $63 \%$, while $25.8 \%$ were partially immunized and $11.3 \%$ of the population studied were not immunized at all according to the NPI schedule.
- $\quad$ From the study 355 (88.9\%) children had BCG vaccine, out of which only $75.8 \%$ had evidence of a BCG scar,while the remaining $24.2 \%$ had no scar.

- $\quad$ Of children delivered in the hospital, majority (72.9\%) were fully immunized compared to children delivered at home in which only $35.2 \%$ were fully immunized.

- Majority (79\%) of caregivers interviewed had knowledge on the NPI schedule as $62.3 \%$ knew that immunization should start immediately after birth. Among those who had knowledge, 51.3\% got their information from radio/television and 34.8\% from health facilities.

- The respondents showed positive attitude towards immunization of children under five, except that most $(80.3 \%)$ are of the view that immunization protects against all forms of diseases.

- The percentage of mothers who completed their TT vaccine was $20.4 \%$, while two - third of the women that received TT had at least TT3.

\section{Recommendations}

Based on the above observations and conclusions, we make the following recommendations;

1. Government should consolidate efforts to accelerate and maintain immunization coverage by an intensive public enlightenment on the importance of immunization as well as strict adherence to the immunization schedule.

2. Emphasis should be made by the federal government on the regular supply of vaccines, good storage and transport facilities, provision and maintenance of basic training programmes for medical and paramedical personnels. This can bring about an increase in the coverage rate.

3. The state and local government should implement programs that will encourage hospital delivery, since hospital delivery has been found to be associated with high immunization coverage rate.

4. The possibility of legislation regarding vaccination certificate being practised in some countries should be adopted in Nigeria by the federal government.

\section{Acknowledgements}

The authors are grateful to the anonymous reviewers for their insightful comments. The authors further wish to acknowledge all those who facilitated the study, too numerous to mention.

\section{REFERENCES}

[1] World Health Organization, “Global Alliance for vaccines 
and immunization (GAVI),” World Health Organization Media Center, 2004. Online available from: http://www.who.int/mediacentre/factsheets/fs169/en/.

[2] J. C. Azubuike and K. E. O. Nkanginieme, Paediatrics and child health in a tropical region. Enugu, Nigeria: Fourth Dimension Publishers, 2007.

[3] World Health Organization, "Immunization, vaccines and Biologicals," 2007. Online available from: http://www.who.int/immunization/en/.

[4] Z. Bhutta et al., "Maternal and child health: is South Asia ready for change?,” $B M J$, vol. 328, no. 7443, pp. 816-819, 2004.

[5] “Nigeria under 5 mortality rate per 1000," Trading Economics. Online available from: www.tradingeconomics.com/nigeria/mortality-rate-under-f ive-per-1,000-wb-data.html.

[6] "The statistic portal," Statista. Online available from: https://www.statista.com/statistics/264714/countries-with-t he-highest-infant-mortality-rate/.

[7] E. A. Ophori, M. Y. Tula, A. V. Azih, R. Okojie, and P. E. Ikpo, "Current Trends of Immunization in Nigeria: Prospect and Challenges,” Trop. Med. Health, vol. 42, no. 2, pp. 6775, 2014.

[8] "The history of vaccines." Online available from: https://www.historyofvaccines.org/content/articles/differen t-types-vaccines.
[9] A. Boylston, “The origins of inoculation,” J. R. Soc. Med., vol. 105, no. 7, pp. 309-313, 2012.

[10] H. Tadesse, A. Deribew, and M. Woldie, "Predictors of defaulting from completion of child immunization in south Ethiopia, May 2008 A case control study,” BMC Public Health, vol. 9, pp. 4-9, 2009.

[11] R. F. Breiman, P. K. Streatfield, M. Phelan, N. Shifa, M. Rashid, and M. Yunus, "Effect of infant immunisation on childhood mortality in rural Bangladesh: analysis of health and demographic surveillance data.," Lancet (London, England), vol. 364, no. 9452, pp. 2204-11, 2004.

[12] National Population Commission \& ICF Macro, "Nigeria Demographic and Health Survey,” 2009. Online available from: https://www.dhsprogram.com/pubs/pdf/FR222/FR222.pdf.

[13] S. Dhanawade, S. Kumbhar, A. Gore, and V. Patil, "Scar formation and tuberculin conversion following BCG vaccination in infants: A prospective cohort study,” J. Fam. Med. Prim. Care, vol. 4, no. 3, p. 384, 2015.

[14] I. B. Ahluwalia, S. D. Helgerson, and F. J. Bia, "Immunization coverage of children in a semi-urban village panchayat in Nepal, 1985," Soc. Sci. Med., vol. 26, no. 2, pp. 265-268, 1988.

[15] M. Naeem et al., "Coverage and factors associated with tetanus toxoid vaccination among married women of reproductive age: a cross sectional study in Peshawar," $J$ Ayub Med Coll Abbottabad, vol. 22, no. 3, pp. 136-140, 2010. 